\title{
Some advances in modeling multiphysics-biomedical applications
}

\author{
Nagi Elabbasi ${ }^{\mathrm{a}}$, Klaus-Jürgen Bathe ${ }^{\mathrm{b}, *}$ \\ ${ }^{a}$ ADINA R\&D, Inc., 71 Elton Avenue, Watertown, MA 02472, USA \\ ${ }^{b}$ Department of Mechanical Engineering, Massachusetts Institute of Technology, 77 Massachusetts Avenue, Cambridge, MA 02139, USA
}

\begin{abstract}
The finite element analysis of biomedical applications requires powerful solid, fluid and multiphysics capabilities. In this paper, a brief summary of some recent multiphysics developments is provided, followed by three demonstrative examples. The first is an illustrative multiphysics problem showing fluid-structure interaction (FSI), flow through porous media, thermal coupling and mass transfer. The second example is a coupled FSI problem involving blood flow through a stenotic artery. The third example involves a helmet impact simulation.
\end{abstract}

Keywords: Multiphysics; Biomedical applications; Fluid-structure interaction; FSI; ADINA

\section{Introduction}

During recent years there has been an increasing interest in the numerical solution of biomedical problems $[1,2]$. Typical problems analyzed are found in the areas related to hemodynamics, artificial organs, orthotics, prosthetics, medical devices, cell mechanics, bioreactors, cartilage/bone mechanics, eye surgery, crash testing, ablation procedures, and drug delivery systems. For the analysis of many such problems multiphysics finite element solution capabilities are needed.

The objective of this paper is to highlight some new finite element multiphysics capabilities that we have been developing, and to provide examples to demonstrate these capabilities [3-5].

\section{Features needed for biomedical multiphysics applications}

Frequently, in biomedical applications fluid-structure interaction (FSI) capabilities are needed. The FSI capabilities relevant to the biomedical field include coupling along solid-fluid boundaries, which is essential for modeling blood circulation problems such as those involving arteriosclerosis, aneurysms, grafts, endovascular stents, or heart valves [6-8]. The coupling between fluids and solid porous media where both the solid and fluid models share

\footnotetext{
* Corresponding author. Tel.: +1 (617) 253-6645; Fax: +1 (617)
} 253-2275; E-mail: kjb@mit.edu the same space is also relevant. This feature is useful in modeling bones and brain tissue. Thermo-mechanical coupling is another multiphysics capability that is needed in biomedical applications such as in eye surgery simulation and ablation procedures [9].

While each physical component in the analysis of a multiphysics problem may be represented by its own different finite element type, mesh topology, and numerical integration procedure, they should all contribute to a single coupled system of equations. And then it is beneficial to have both direct and iterative solution algorithms for the coupled system of equations. The iterative solution algorithm can use existing fluid and structural solvers. However, for problems involving strong coupling a direct solver is required.

Regarding the modeling of the kinematics and material behavior, the accurate simulation of biomedical problems requires powerful $2 \mathrm{D} / 3 \mathrm{D}$ solid elements, beam and shell elements, with linear and nonlinear responses and an extensive material library. The material library should include several hyperelastic models suitable for blood vessels, viscoelastic models suitable for cells, and porous models suitable for biological tissues. A robust frictional contact algorithm is required for numerous biomedical problems such as encountered in nerve contact, orthotic components and spine mechanics [10-12]. The fluids module of a computational code should be able to handle the Navier-Stokes incompressible, slightly compressible and low-speed compressible flows. It should handle mass transfer equations, which are useful in simulating drug delivery problems or bioreactors [13]. It should also include Poisson-type equa- 
tions for electrical potentials and displacements, which are needed for modeling tissue ablation [9]. Finally, powerful pre- and post-processing features and CAD support are necessary in order to provide users with a versatile user-friendly environment.

\section{Demonstrative examples}

In this section we present several biomedical-related examples. The simulations were performed using the ADINA System [14], which has the above-mentioned multiphysics capabilities. More case studies can be found in Refs. [6-13].

\subsection{Illustrative multiphysics problem}

This example is constructed to demonstrate several multiphysics features in a single model. It involves a transient analysis of a fluid flowing past two thin flexible solid walls, as shown in Fig. 1. The lower wall is assumed to be porous. The fluid at the inlet is subjected to a pressure rise that propels it past the flexible walls. A prescribed temperature rise is applied to the solid at the inlet side, and prescribed mass concentration is also imposed at a fluid point. This example involves fluid-structure coupling, (i) along a fluid-structure interface, (ii) resulting from thermal coupling, and (iii) resulting from porous media. The example also involves mass transfer analysis.

Fig. 1 also shows some results from this model that illustrate the deformations of the structure due to the fluid pressure and the non-symmetry in the flow due to the porous wall. Note that the fluid-flow with mass transfer features used in this example can be employed to study the delivery of drugs through the bloodstream, while the fluid flow across porous and non-porous walls can be used, for example, to study blood flow in the lungs and across heart valves.

\subsection{Blood flow through a stenotic artery}

Blood circulation and other hemodynamics problems can most accurately be modeled as coupled FSI problems. The blood vessels are generally compliant, and their deformations affect the flow of the blood. In this example, we present a simulation of the stenotic (narrow or constricted) artery shown in Fig. 2. A severe stenosis is selected where the internal diameter of the artery is reduced by $80 \%$. Under these conditions the artery may experience high shear stresses and blood recirculation [7].

The artery is modeled as a hyperelastic material undergoing large deformations and the blood as a Newtonian fluid. Stiffer material properties are assumed in the vicinity of the stenotic section. An Arbitrary Lagrangian Eulerian (ALE) formulation is used for the fluid mesh to minimize mesh distortion as the artery gets stretched. The artery is first subjected to an axial stretch of $30 \%$ to simulate its in vivo condition. This leads to a reduction in its internal diameter. Blood is then pumped through the artery resulting in its expansion. This is followed by several pressure cycles emulating the pressure variation during circulation. The direct computing method is used to solve the single effective stiffness matrix resulting from the coupled problem. Further details on the solution procedure for coupled problems can be found in Refs. [5,9,14].

Fig. 2 also shows the pressure variation in the blood, and the axial strain in the artery at a certain time during the cyclic part of the simulation. The results illustrate the high axial strain in the artery and the severe pressure drop at the constricted section.
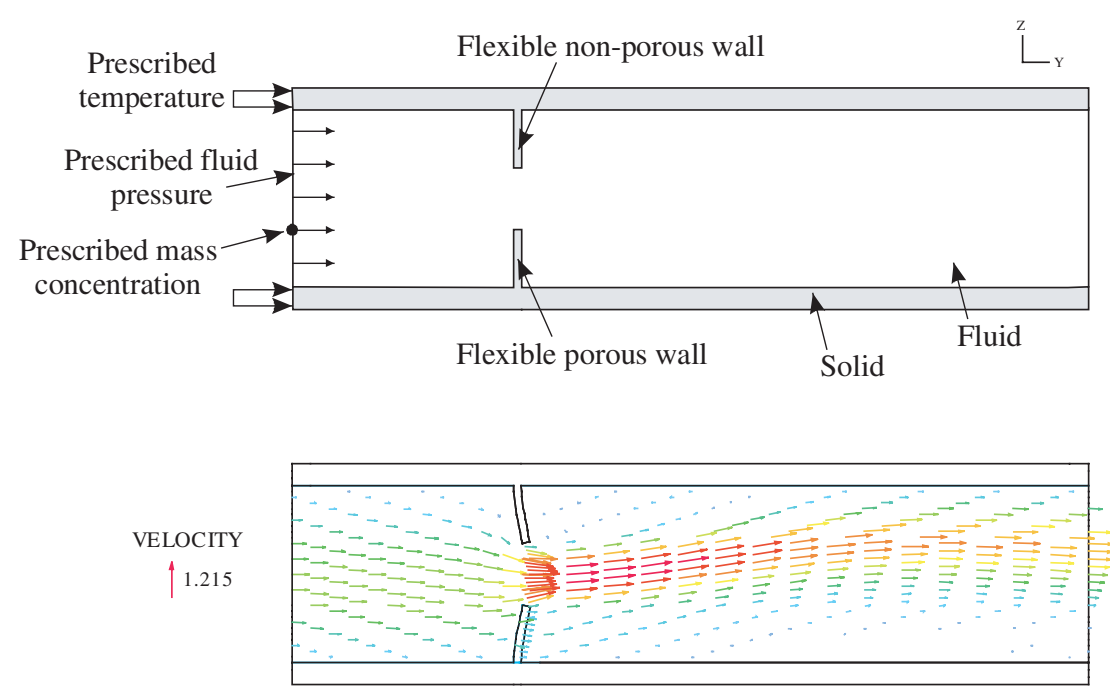

Fig. 1. Illustrative multiphysics problem: schematic (top) and velocity profile (bottom). 


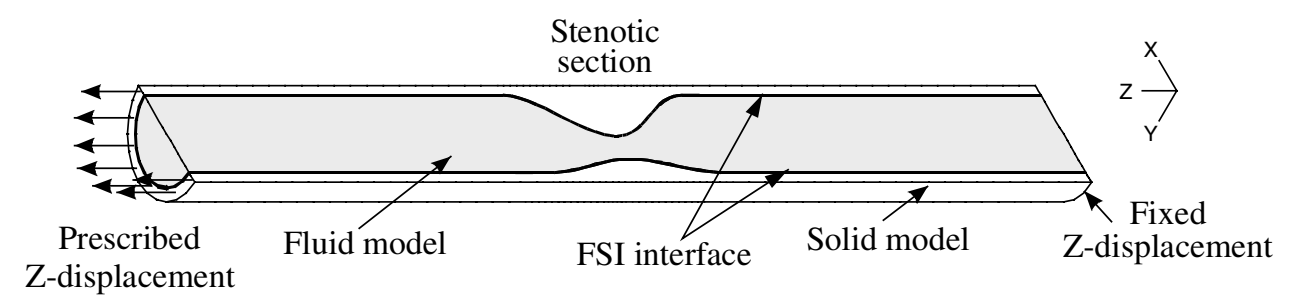

Z-displacement

SMOOTHED STRAIN-ZZ

$-0.4200$

$-0.3600$

-0.3000
-0.2400

-0.2400
-0.1800
-

$-0.1200$

0.0600

$\triangle 0.4218$

MINIMUM
SMOOTHED FE_PRESSURE

$\begin{array}{ll} & -108000 . \\ & =90000 . \\ \text { MAXIMUM } & -72000 . \\ \triangle 111751 . & -54000 . \\ \text { MINIMUM } & -18000 . \\ * 4651 . & -0 .\end{array}$

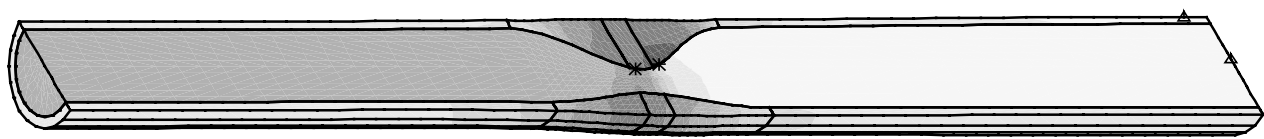

Fig. 2. Stenotic artery model (top) and fluid pressure and solid strain-zz distribution (bottom).
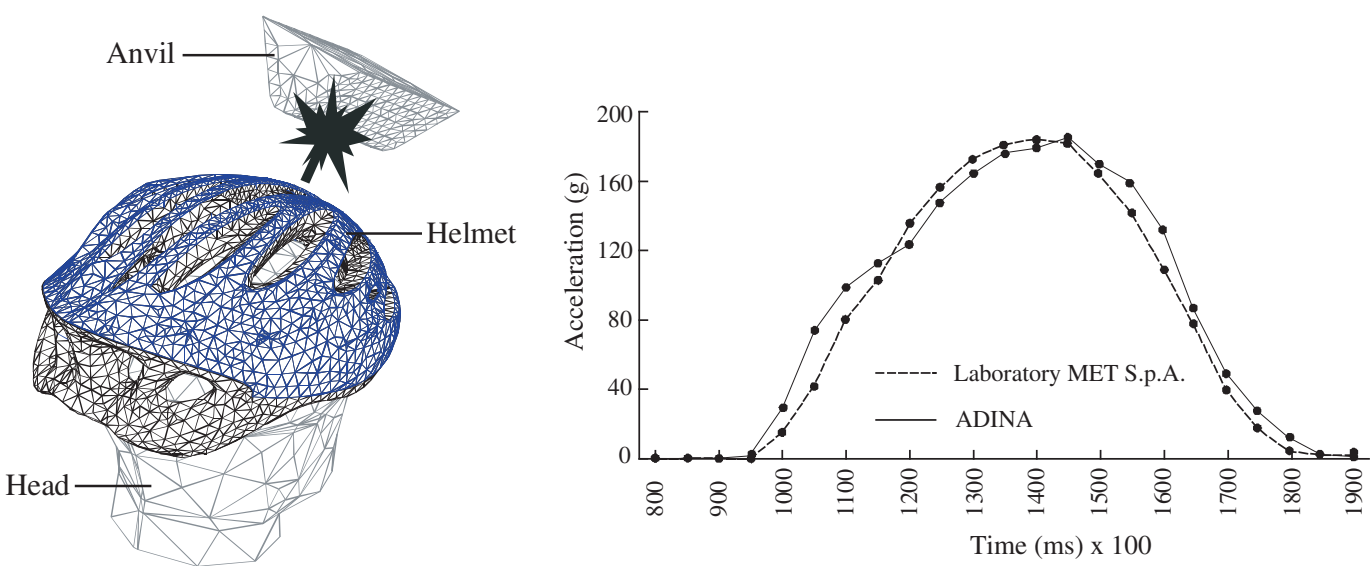

Fig. 3. Helmet impact analysis: schematic (left), and comparison with experimental data (right).

\subsection{Helmet impact analysis}

Sports helmets are essential for mitigating head and neck injury. Fig. 3 shows a finite element model of a new helmet design (courtesy of MET S.p.A., Italy). The objective of the analysis was to ensure that the helmet provides adequate protection for the head. In particular, the deceleration of the head must not exceed $250 \mathrm{~g}$. Experimental tests were also carried out on prototype models in a laboratory setting.

The helmet was modeled using 26,800 3D elasto-plastic solid elements and the head was given an initial velocity of $4.57 \mathrm{~m} / \mathrm{s}$. The anvil was modeled using 3D elastic elements with material properties corresponding to steel. An implicit dynamic simulation was performed, of course, without any reduced integration, hourglass control or mass scaling. Fig. 3 also compares the acceleration versus time curves obtained from the numerical simulation and laboratory test results. There is very good agreement between the simulation results and the experimental data.

\section{Concluding remarks}

The biomedical field provides for exciting research developments and applications in computational mechanics. In this paper, we have briefly addressed some developments in finite element methods for the multiphysics problems encountered. But, of course, further enhancements are much needed and we are continuing our developments.

\section{References}

[1] Bathe KJ (Ed). Computational Fluid and Solid Mechanics: Proc First MIT Conference on Computational Fluid and Solid Mechanics. Elsevier Science, 2001. 
[2] Kaazempur-Mofrad MR, Bathe M, Karcher H, Younis HF, Seong HC, Shim EB, Chan RC, Hinton DP, Isasi AG, Upadhyaya A, Powers MJ, Griffith LG, Kamm RD. Role of simulation in understanding biological systems. Computers and Structures (in press).

[3] Bathe KJ. Finite element procedures. New York: Prentice Hall, 1996.

[4] Rugonyi S, Bathe KJ. On finite element analysis of fluid flows fully coupled with structural interactions. Computer Modeling in Engineering and Sciences 2001;2:195-212.

[5] Bathe KJ, Zhang H, Ji S. Finite element analysis of fluid flows fully coupled with structural interactions. Computers and Structures 1999;72:1-16.

[6] Rogers C, Tseng DY, Squire JC, Edelman ER. Balloonartery interaction during stent placement. A finite element analysis approach to pressure, compliance, and stent design as contributors to vascular injury. Circulation Research 1999;84: 378-383.

[7] Tang D, Yang C, Zheng J, Vito RP. Stress/strain analysis of arteries with stenotic plaques and lipid cores. Proc Fourth World Congress of Biomechanics, 2002.

[8] Bathe M, Shirai A, Doerschuk CM, Kamm RD. Neutrophil transit times through pulmonary capillaries: The effects of capillary geometry and fMLP-stimulation. Biophysical J 2002;83: 1917-1933.

[9] Zhang H, Zhang X, Ji S, Guo Y, Ledezma G, Elabbasi $\mathrm{N}$, deCougny $\mathrm{H}$. Recent development of fluid-structureinteraction capabilities in the ADINA System. Computers and Structures (in press).

[10] Petrie CP, Brown TD, Steyers CM. Finite element analysis of median nerve insult in the carpal tunnel. Proc Fourth World Congress of Biomechanics, 2002.

[11] Chu TM, Reddy NP. Stress distribution in the ankle-foot orthosis used to correct pathological gait. J Rehabilitation Research and Development 1995;32:349-360.

[12] Natarajan RN, Andersson GBJ. The influence of lumbar disc height and cross-sectional area on the mechanical response of the disc to physiologic loading. Spine 1999;24: 1873-1881.

[13] Kaazempur-Mofrad MR, Sivaraman A, Upadhyaya A, Domansky K, Powers MJ, Kamm RD, Griffith LG. Computational modeling of flow and mass transport in a microfabricated array bioreactor for perfused 3-D liver culture. Proc Fourth World Congress of Biomechanics, 2002.

[14] ADINA R \& D, Inc. ADINA Theory and modeling guide. Vols. I, II, III. Watertown, MA, 2002. 